OPEN ACCESS

Edited by:

Jan A. Plock,

Aarau Cantonal Hospital, Switzerland

Reviewed by:

Fatih Zor,

Wake Forest School of Medicine,

United States

Ravi Kumar Chittoria, Jawaharlal Institute of Postgraduate

Medical Education and Research

(JIPMER), India

*Correspondence:

Luke Taylor

luke.taylor2@uhbw.nhs.uk

Specialty section:

This article was submitted to Reconstructive and Plastic Surgery,

a section of the journa

Frontiers in Surgery

Received: 05 August 2021 Accepted: 11 November 2021

Published: 10 December 2021

Citation:

Taylor L, Asmar O, Mandal A, Tridente A, Hardy K and Shokrollahi K (2021) Perspectives From a Regional

Plastic Surgery Centre on Evidence for the Purported Link Between SGLT2 Inhibitors and Fournier's Gangrene.

Front. Surg. 8:754101.

doi: 10.3389/fsurg.2021.754101

\section{Perspectives From a Regional Plastic Surgery Centre on Evidence for the Purported Link Between SGLT2 Inhibitors and Fournier's Gangrene}

\author{
Luke Taylor $^{1 *}$, Omar Asmar ${ }^{2}$, Anirban Mandal ${ }^{2}$, Ascanio Tridente ${ }^{3}$, Kevin Hardy $^{4}$ and \\ Kayvan Shokrollahi ${ }^{2}$
}

${ }^{1}$ Department of Upper Gastrointestinal Surgery, Royal Gwent Hospital, Newport, United Kingdom, ${ }^{2}$ Mersey Regional Centre for Burns and Plastic Surgery, Whiston Hosiptal, Liverpool, United Kingdom, ${ }^{3}$ Department of Critical and Intensive Care, Whiston Hospital, Liverpool, United Kingdom, ${ }^{4}$ Department of Diabetology and Endocrinology, Whiston Hospital, Liverpool, United Kingdom

Introduction: The recent report issued by the MHRA indicating an association of Sodium glucose linked transporter type 2 (SGLT2) Inhibitors with the contraction of Fournier's Gangrene (FG), has been drawn with insufficient supporting evidence and without an adequately powered study to make any meaningful assertions or recommendations. We aimed to look specifically at the currently available dataset used to link SGLT2 Inhibitors to FG and highlight what conclusions or inferences can meaningfully be made, in particular the power of any study that would be required to make sensible conclusions.

Methods: World literature review of SGLT2 Inhibitors and FG was performed. With a subsequent 10-year review of cases of FG seen in a regional burns and plastics centre. Data was collected retrospectively from the coding department at Whiston Hospital for all patients with necrotising fasciitis. An electronic document management system was used to identify patients with FG specifically as well as their diabetes state and medication history.

Results: Seventy-eight patients were admitted with FG, of whom 32 had diabetes mellitus (DM). Of those with DM none was taking an SGLT2 Inhibitor, 17 patients were taking metformin, a further nine patients were taking a second line medication and 14 required insulin injections.

Discussions: DM is a known major risk factor for $F G$, which is clearly observed in our patient cohort. The risk of patients with DM developing FG is irrespective of the medication patients are taking. The current articles and reports published have little ground to claim an association between SGLT2 Inhibitors and FG and are missing the crucial message that needs to be conveyed to the public: that DM is a major risk factor for FG and patients suffering with diabetes need to be extra vigilant.

Keywords: burns-pathology, surgery, reconstructive surgery, Fournier's Gangrene, SGLT2 inhibitors, plastic surgery, type 2 diabetes (T2D) 


\section{INTRODUCTION}

Sodium glucose linked transporter type 2 (SGLT2) largely mediates the reabsorption of glucose in the proximal tubules of the kidneys (1). Inhibition of SGLT2 leads to an increase in glucose excretion via the kidneys as well as a reduction in the levels of plasma glucose, all independently of insulin (2). Pharmacological agents that block the actions of SGLT2, termed SGLT2 Inhibitors, have recently become available for the management of Type 2 Diabetes Mellitus (T2DM) (3). Research into SGLT2 Inhibitors began back in the early 1980's with the first approval for use in treatment of type 2 diabetes mellitus (T2DM) in 2013 (4). There are four SGLT2 inhibitors currently licenced for use in the UK; Dapagliflozin, Empagliflozin, Canagliflozin and Ertugliflozin, as well as combinations of these drugs with other diabetic medications, see also Table 1 (5). These novel drugs have been associated with improved cardiovascular risk factors and slower progression of renal disease in diabetic patients, as well as promoting some weight loss $(6,7)$. However, they do have some common side effects, including urinary tract and genital infections; which patients with diabetes are already at increased risk of developing $(8,9)$.

The pathophysiology of T2DM puts patients at greater risk of developing urinary tract and genital infection. Factors such as glucosuria, immune dysfunction, raised oestrogen levels and increased adherence of bacteria to uroepithelium all contribute to this increased risk $(10,11)$. As SGLT2 Inhibitors lead to increased glucose excretion via the kidneys, exacerbating the pathophysiology that occurs in the urinary tract of a diabetic patient, it would be expected that there would be an increased incidence of genitourinary infections (12-14). In rare cases diabetic patients can develop a severe infection known as Fournier's Gangrene, a fulminant form of necrotising fasciitis affecting the groin, perineum and genital areas $(15,16)$. This condition can be life threatening and requires immediate surgical excision and debridement of infected tissue, with likely reconstruction needed in a specialist centre (17).

In recent months, the Medicines and Healthcare Products Regulatory Agency (MHRA) and the Food and Drug Administration Agency (FDA) have issued reports indicating an association of SGLT2 inhibitors with Fournier's Gangrene contraction (18-20). The MHRA has published that it has received six yellow card warnings (21) of SGLT2 Inhibitors associated with Fournier's Gangrene; four men and two women (19). While the FDA reported 12 recorded cases of SGLT2 Inhibitors associated with Fournier's Gangrene over a period of March 2013 to May 2018 (18, 22-24). These results are shown in Table 2. These reports are describing a possible causative link between patients taking SGLT2 Inhibitors and Fournier's Gangrene, however, there is no statistical evidence to support a correlation between the two variables. The overarching combined message from the MHRA and FDA is that of a warning

Abbreviations: SGLT2, Sodium glucose linked transporter type 2; FG, Fournier's Gangrene; DM, Diabetes Mellitus; T2DM, Type 2 Diabetes Mellitus; MHRA, Medicines and Healthcare Products Regulatory Agency; FDA, Food and Drug Administration Agency; EDMS, Electronic Document Management System.
TABLE 1 | List of SGLT2 inhibitors currently licenced for use in the UK according to the NICE clinical guidelines.

\begin{tabular}{ll}
\hline Drug name & Brand name \\
\hline Dapagliflozin & Forxiga, Edistride \\
Canagliflozin & Invokana \\
Empagliflozin & Jardiance \\
Ertugliflozin & Steglatro \\
Dapagliflozin/Metformin & Ebymect \\
Dapagliflozin/Metformin & Xigduo \\
Dapagliflozin/Saxagliptin & Qtern \\
Canagliflozin/Metformin & Vokanamet \\
Empagliflozin/Metformin & Synjardy \\
Empagliflozin/Linagliptin & Glyxambi \\
Ertugliflozin/Metformin & Segluromet \\
Ertugliflozin/Sitagliptin & Steglujan \\
\hline
\end{tabular}

Brand names for each drug have also been listed (5).

TABLE 2 | The number of cases of Fournier's Gangrene associated with the use of SGLT2 inhibitors reported from both the Medicines and Health Regulatory Agency and the Food and Drug Administration Agency.

\begin{tabular}{lcccl}
\hline No. cases & No. men & No. women & $\begin{array}{l}\text { SGLT2 inhibitors } \\
\text { associated }\end{array}$ \\
\hline MHRA & 6 & 4 & 2 & Not reported \\
FDA & 12 & 7 & 5 & $\begin{array}{l}\text { Dapagliflozin, } \\
\text { Canagliflozin, } \\
\text { Empagliflozin }\end{array}$ \\
\hline
\end{tabular}

to both patients and clinicians. The warning to patients states that upon experiencing symptoms of tenderness, redness or swelling of the genitals and surrounding area as well as having an associated fever they should seek immediate medical attention. The message to health care professionals states that any patient with the above described symptoms should be examined for Fournier's Gangrene and, if taking an SGLT2 Inhibitor, should be stopped immediately $(18,20)$.

Since the initially published warning by the FDA, it subsequently conducted a descriptive cases series over from the time frame of 1 March 2013 to the 31 January 2019. The results from this study are shown in Table 3 (25). The study describes an additional 35 cases of FG in association with an SGLT2 Inhibitor from the initial 12 cases that prompted the warning in February 2019. The study also identifies a further 19 cases of FG that are associated with other diabetic medications. It is also worth noting that the study highlights the limitation of the inability to establish causality or incidence.

Over 500,000 patients are currently estimated to have exposure to an SGLT2 Inhibitor in the UK (26), and $\sim 1.7$ million patients have exposure in the USA $(18,27)$. This represents a vast population size of $\sim 2.2$ million people, yet only 18 cases of Fournier's Gangrene have been published with an association to patients taking an SGLT2 Inhibitor, far below the numbers required to prove causation between two independent variables. 
TABLE 3 | Results of a descriptive case series carried out by the FDA in June 2019 after the initially published warning in February 2019 (25).

\begin{tabular}{|c|c|c|c|c|c|c|}
\hline No. cases & No. men & No. women & Age & Time on SGLT2 inhibitor & No. requiring surgery & No. complications \\
\hline 55 & 39 & 16 & $33-87$ & 5 days -49 months & 55 & 21 \\
\hline
\end{tabular}

TABLE 4 | Showing the percentages of patients with DM taking an SGLT2 inhibitor and the percentage of patients taking other 2nd line therapy for control of their diabetes.

\begin{tabular}{|c|c|c|c|c|c|}
\hline Number of patients & $\%$ of patients with DM & $\begin{array}{l}\text { \% DM patients } \\
\text { taking SGLT2 } \\
\text { inhibitor }\end{array}$ & $\begin{array}{c}\text { \% DM patients taking } \\
\text { other } 2 \text { nd line DM } \\
\text { medication }\end{array}$ & $\begin{array}{l}\% \text { DM patients } \\
\text { taking metformin }\end{array}$ & $\begin{array}{l}\text { \% DM patients } \\
\text { taking insulin }\end{array}$ \\
\hline 78 & 41.02 & 0 & 28.13 & 53.13 & 43.75 \\
\hline
\end{tabular}

There have been no other studies to show any association between the diabetes medication patients are taking with the development of Fournier's Gangrene. Diabetes itself is the major risk factor for contraction of Fournier's Gangrene $(11,16)$ and demonstrating or refuting a link between any specific medication is not possible without a very large sample size and comparison with other medications.

The vast majority of patients with Fournier's Gangrene across the North West of England and Wales are transferred to Whiston Hospital in order to receive specialist treatment and reconstruction by the regional Burns and Plastics Department. The aim of this study was to retrospectively analyse all patients with Fournier's Gangrene seen at a hospital with a regional burns and plastic service over the last 10 years, reviewing the specific diabetic medications each patient was taking prior to their admission, and to explore further reports of this potential link.

\section{METHODS}

\section{Literature Review}

A review of world literature on the subjects of SGLT2 Inhibitors and Fournier's Gangrene was performed. With a subsequent 10year review of cases of Fournier's Gangrene seen in a regional burns and plastics centre.

\section{Data Collection}

A database of all patients coded for Necrotising Fasciitis admitted into Whiston Hospital between January 2008 and December 2018 was retrospectively generated. An electronic document management system (EDMS) was used to review the notes for each patient in order to collate only the patients with Fournier's Gangrene and also to identify their diabetes state. EDMS was also used to analyse the medication history of each patient to identify patients taking an SGLT2 Inhibitor, as well as all other diabetic medications.

\section{Data Analysis}

Data was analysed using JMP ${ }^{\circledR}$ statistical software and GraphPad Prism $8^{\circledR}$ software was used to generate and edit all graphs.

\section{RESULTS}

Analysis of patient data showed that 78 patients were admitted to Whiston Hospital, either with Fournier's Gangrene or for surgical reconstruction post-debridement following an episode of Fournier's Gangrene between January 2008 and December 2018. Of these patients a total of 32 had diabetes mellitus (DM), either type 1 or type 2 . The age range for these patients was 31-97 years old, with the median age being 64 (lower and upper interquartile ranges are 54 and 78 respectively).

As is seen in Table 2, 41.02\% $(n=32)$ of patients with Fournier's Gangrene also had DM, of whom all were on medication in order to control their blood glucose levels. As per NICE guidelines some patients were taking more than one medication in order to control their diabetes. As shown in Table 4, 53.13\% $(n=17)$ of patients were taking metformin, with a further $28.13 \%(n=9)$ of patients requiring another second line medication to reach adequate diabetes control; breakdown of which medications were taken is shown in Figure 1. It was observed that no patients admitted with Fournier's Gangrene were taking an SGLT2 Inhibitor.

Figure 1A shows the number of patients presenting with Fournier's Gangrene who had DM (32 patients) and those who did not (46 patients). Figure 1B shows the breakdown of diabetes medication taken by the 32 patients with DM. Seventeen patients were taking metformin with a further nine patients requiring a second line treatment as well, either gliclazide or sitagliptin. A total of 14 patients required insulin for their diabetes control, either as primary treatment for T1DM (11 patients) or for add on therapy for T2DM (three patients). No patients were taking an SGLT2 Inhibitor for control of their diabetes.

\section{DISCUSSION}

Diabetes UK currently estimate that 4.6 million people are living with diabetes in the UK, $90 \%$ of whom are T2DM, with an increasing number of patients being diagnosed each year (28). There are $\sim 500,000$ patients in the UK currently taking an SGLT2 Inhibitor for diabetes control, therefore it stands that $\sim 12 \%$ of all T2DM patients in the UK are taking an SGLT2 Inhibitor (26). Thus, it would be expected that approximately three to four patients in our dataset were taking an SGLT2 Inhibitor, 
A

\section{Diabetes vs No Diabetes}

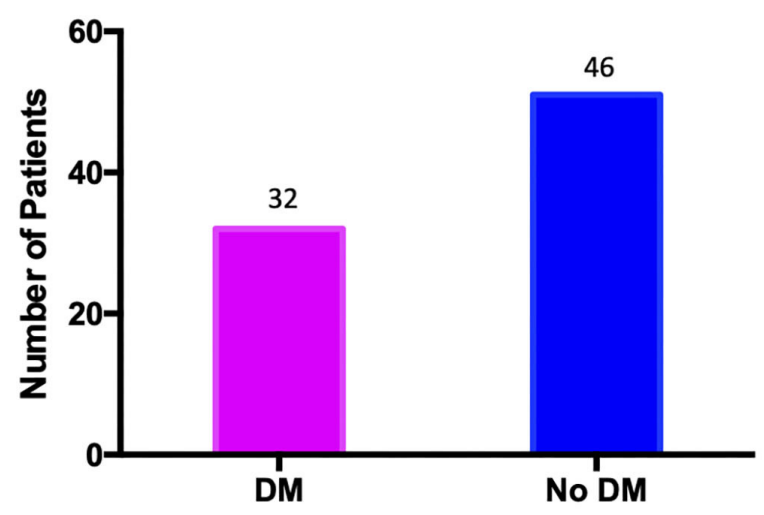

B

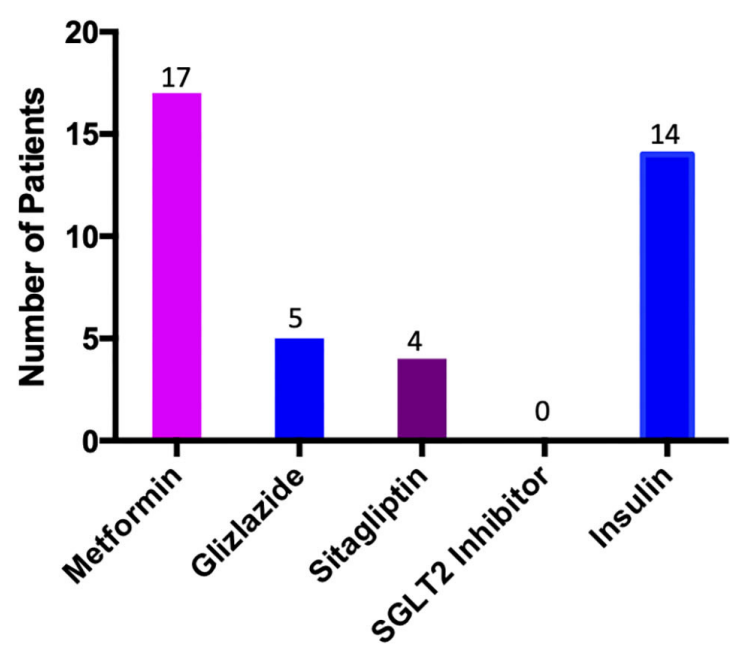

FIGURE 1 | Graphs to show (A) the number of patients presenting with Fournier's Gangrene who had diabetes mellitus (DM) against the number who did not (No DM); (B) the number of patients with diabetes mellitus taking each individual medication.

however it was observed that none of the patients presenting with Fournier's Gangrene were taking an SGLT2 Inhibitor upon their admission into hospital.

Fournier's Gangrene is rare, and while we did not find any patients taking an SGLT2 Inhibitor who developed Fournier's, we did discover that they were taking a range of other diabetic medication. There is currently insufficient evidence in world literature to either support or refute an association of SGLT2 Inhibitors, or any diabetic medication in fact, with development of Fournier's Gangrene. It is clear, however, that the size of the study required to show correlation is undeniably large and will require collection of data on a national scale. Diabetes mellitus itself, rather than SGLT2 Inhibitors, is the major risk factor for development of Fournier's Gangrene, as supported by data from our own study with over $40 \%$ of patients having diabetes at the time of their hospital admission. It is known that diabetes mellitus predisposes patients to an increased risk of urinary tract and genital infections due to raised urine glucose content as well as reducing host immune factors $(29,30)$. When comparing SGLT2 Inhibitors to other second line T2DM medications, it was observed that there was no difference in the risk of urinary tract and genital infections between the two cohorts (31). These data suggest that all T2DM patients are at equal risk of developing these infections if their diabetes control is not adequate, irrespective of the specific medications they are taking. In fact, $28.13 \%$ of patients with T2DM who were admitted to Whiston Hospital with Fournier's Gangrene were requiring a second line medication (gliclazide or sitagliptin) on top of metformin, the first line medication given in T2DM as recommended by NICE (5). The requirement for second line diabetic medication in T2DM, such as an SGLT2 Inhibitor, therefore, could be a surrogate marker of poorly controlled diabetes. However, accurate $\mathrm{HbA1c}$ data would be required to show whether the significant risk of T2DM in patients developing Fournier's Gangrene appears to be poorly controlled diabetes rather than simply diabetes itself.

The only published negative effects of SGLT2 Inhibitors that have a statistical significance are the increased risk of diabetic ketoacidosis (DKA) and lower digital amputation, published in separate studies including over 17,000 patients currently taking an SGLT2 Inhibitor $(31,32)$. The current association between SGLT2 Inhibitors and Fournier's Gangrene published by the MHRA and FDA do not demonstrate correlation. This is due, in part, to the extremely small patient cohort and number of confounding variables. The suggested reports of SGLT2 inhibitors causing Fournier's Gangrene is at odds not only with our experience but the underlying case numbers and power of studies and reports simply cannot reasonably lead to the conclusions made. In order to do such would require a study size similar to that required for a phase 3 clinical trial of any pharmaceutical agent $(33,34)$. We have tentatively calculated the estimated sample size which may be required. The calculations make the following assumptions: we use the incidence in the male diabetic population of $1.6 / 100,000 /$ year and we assume that a significant increase in the incidence in this population related to the use of SGLT2 Inhibitors would be $20 \%$, increasing it to $1.92 / 100,000 /$ year (male). We assume $10 \%$ of the sample would be lost to follow-up and 10\% in each arm would cross over to the other arm. Assuming a 1:1 randomisation ratio, observation of 125,325,522 person-years would be required across the two arms (35). If we aimed to detect an increase in incidence rate of just $10 \%$, all other assumptions remaining the 
same, observation of $478,515,626$ person-years would be required across the two groups.

Our hospital data reconfirms diabetes mellitus as a significant risk factor for Fournier's Gangrene, with over $40 \%$ of patients having diabetes. Our study demonstrated that no diabetic patients in a regional centre with a catchment area of over five million patients who were admitted with Fournier's Gangrene were taking an SGLT2 Inhibitor. It is important to emphasise that this study in no way proves the absence of a link between Fournier's Gangrene and SGLT2 Inhibitors in exactly the same way that the studies already published suggestive of a link do not actually prove such a link exists $(18,19,21-24)$. Our view is that in the absence of sufficiently powered studies the message from the MHRA is not that "cases of Fournier's Gangrene have been associated with SGLT2 Inhibitors and patients should seek urgent medical attention if experiencing any of the described symptoms" (36), rather the message should read "patients with diabetes mellitus in general, particularly if poorly controlled, should be extra vigilant to the symptoms of Fournier's Gangrene and indeed necrotising fasciitis anywhere in the body." This broader vigilance still protects the SGLT2-taking diabetic population until such time as any causal relationship is proven with sufficiently powered studies.

\section{CONCLUSIONS}

Diabetes mellitus (DM) is a major risk factor for Fournier's Gangrene, irrespective of the medication patients are taking. This makes it very difficult to link any diabetic medication to a relatively rare complication (Fournier's gangrene) without an enormous dataset and a sufficiently powered study. This is highlighted by the fact that in our regional service over a 10-year period not a single patient with Fournier's gangrene was taking an SGLT2 Inhibitor prior to their admission.

The recent messaging from MRHA and FDA with regards to risk of Fournier's Gangrene in patients taking SGLT2 inhibitors is more helpfully presented as a general awareness across the board of all diabetic patients and their risk of this devastating condition.

Our appraisal of our own data and what is currently available demonstrates that there is currently no evidence for a causal relationship between SGLT2 Inhibitors and Fournier's Gangrene other than the causal association between diabetes itself and Fournier's gangrene, and the published studies are lacking in power to prove such. In order to refute or prove a causal relationship, adequately powered studies that specifically take

\section{REFERENCES}

1. Hasan FM, Alsahli M, Gerich JE. SGLT2 inhibitors in the treatment of type 2 diabetes. Diabetes Res Clin Pract. (2014) 104:297-322. doi: 10.1016/j.diabres.2014.02.014

2. Gallo LA, Wright EM, Vallon V. Probing SGLT2 as a therapeutic target for diabetes: basic physiology and consequences. Diabetes Vasc Dis Res. (2015) 12:78-89. doi: 10.1177/1479164114561992

3. SGLT2 inhibitors. European Medicines Agency (2019). Available online at: https://www.ema.europa.eu/en/medicines/human/referrals/sglt2-inhibitors (accessed May 24, 2019). into account a patient's diabetes control, the use of diabetic medication (both first- and second-line treatments) as well as patient's compliance with their treatment is required. Our power calculation reveals that a 2-armed study of over 125 million patients would be needed to infer a significant increase in incidence related to exposure to the medications, and this may not be feasible. We are reassured by the absence of any patients in our catchment affected by this suggested link with SGLT2inhibitors. Currently the most important message that needs to be conveyed to the public is that patients suffering with DM need to be extra vigilant for signs of Fournier's Gangrene regardless of their medication.

Robust statistical evidence does not exist that patients taking SGLT2 inhibitors are at greater risk of Fournier's gangrene either (a) because of that medication rather than their diabetes or (b) because of that medication as opposed to other more commonly consumed medications taken by diabetic patients. Prospective studies of considerable magnitude will be needed to demonstrate or refute any such association with any certainty, but it is clear from our appraisal that there appears more room for reassurance than for alarm.

\section{DATA AVAILABILITY STATEMENT}

The raw data supporting the conclusions of this article will be made available by the authors, without undue reservation.

\section{AUTHOR CONTRIBUTIONS}

LT performed data collection and analysis, created the figures, and was the primary author in writing the paper. OA performed data collection. AM provided feedback and contributed improvements for the design and write up of the paper. AT conducted statistical analyses for the paper and provided the power calculation required for future studies. $\mathrm{KH}$ provided expertise into the diagnoses of diabetes as well as the management and control of diabetes with regards to the medications reviewed in the paper. All authors contributed to the article and approved the submitted version.

\section{FUNDING}

Funding for the paper was provided by the burns and plastics research fund for St Helens and Knowsley NHS Trust.

4. Story of Discovery. SGLT2 Inhibitors: Harnessing the Kidneys to Help Treat Diabetes. Bethesda, MD: NIDDK (2019). Available online at: https:// www.niddk.nih.gov/news/archive/2016/story-discovery-sglt2-inhibitorsharnessing-kidneys-help-treat-diabetes (accessed May 24, 2019).

5. BNF: British National Formulary. NICE (2019). Available online at: https:// bnf.nice.org.uk/treatment-summary/type-2-diabetes.html (accessed May 24, 2019).

6. Zinman B, Wanner C, Lachin JM, Fitchett D, Bluhmki E, Hantel S, et al. Empagliflozin, cardiovascular outcomes, and mortality in type 2 diabetes. N Engl J Med. (2015) 373:2117-28. doi: 10.1056/NEJMoa15 04720 
7. Wanner C, Inzucchi SE, Lachin JM, Fitchett D, von Eynatten M, Mattheus M, et al. Empagliflozin and progression of kidney disease in type 2 diabetes. $N$ Engl J Med. (2016) 375:323-34. doi: 10.1056/NEJMoa1515920

8. Immunology Full Video. USMLE Step 1-YouTube (2018). Available online at: https://www.youtube.com/watch? $=$ Mefr4woK19E (accessed May 24, 2019).

9. Highlights of Prescribing Information (2019). Available online at: www.fda.gov/medwatch (accessed May 24, 2019).

10. Achkar JM, Fries BC. Candida infections of the genitourinary tract. Clin Microbiol Rev. (2010) 23:253-73. doi: 10.1128/CMR.00 076-09

11. Benfield T, Jensen JS, Nordestgaard BG. Influence of diabetes and hyperglycaemia on infectious disease hospitalisation and outcome. Diabetologia. (2007) 50:549-54. doi: 10.1007/s00125-006-0570-3

12. Bode B, Stenlöf K, Harris S, Sullivan D, Fung A, Usiskin K, et al. Longterm efficacy and safety of canagliflozin over 104 weeks in patients aged 55-80 years with type 2 diabetes. Diabetes Obes Metab. (2015) 17:294303. doi: $10.1111 /$ dom. 12428

13. Haering H-U, Merker L, Christiansen AV, Roux F, Salsali A, Kim G, et al. Empagliflozin as add-on to metformin plus sulphonylurea in patients with type 2 diabetes. Diabetes Res Clin Pract. (2015) 110:8290. doi: 10.1016/j.diabres.2015.05.044

14. Jabbour SA, Hardy E, Sugg J. Parikh, S, Study 10 Group. Dapagliflozin is effective as add-on therapy to sitagliptin with or without metformin: a 24-week, multicenter, randomized, double-blind, placebo-controlled study. Diabetes Care. (2014) 37:740-50. doi: 10.2337/dc13-0467

15. Thwaini A, Khan A, Malik A, Cherian J, Barua J, Shergill I, et al. Fournier's gangrene and its emergency management. Postgrad Med J. (2006) 82:5169. doi: 10.1136/pgmj.2005.042069

16. Gürlek A, Firat C, Öztürk AE, Alaybeyoglu N, Fariz A, Aslan S. Management of necrotizing fasciitis in diabetic patients. J Diabetes Complications. (2007) 21:265-71. doi: 10.1016/j.jdiacomp.2006.08.006

17. Chawla SN, Gallop C, Mydlo JH. Fournier's gangrene: an analysis of repeated surgical debridement. Eur Urol. (2003) 43:572-5. doi: 10.1016/S0302-2838(03)00102-7

18. Research C for DE and Drug Safety and Availability. FDA Warns About Rare Occurrences of a Serious Infection of the Genital Area With SGLT2 Inhibitors for Diabetes (2019). Available online at: https://www.fda.gov/Drugs/DrugSafety/ ucm617360.htm (accessed May 24, 2019).

19. Medicines and Healthcare Products Regulatory Agency. SGLT2 Inhibitors: Reports of Fournier's Gangrene (Necrotising Fasciitis of the Genitalia or Perineum)-GOV.UK (2019). Available online at: https://www.gov.uk/drugsafety-update/sglt2-inhibitors-reports-of-fournier-s-gangrene-necrotisingfasciitis-of-the-genitalia-or-perineum (accessed May 24, 2019).

20. Drug Safety Update Latest Advice for Medicines Users. The Monthly Newsletter From the Medicines and Healthcare Products Regulatory Agency and Its Independent Advisor the Commission on Human Medicines (2019). Available online at: www.evidence.nhs.uk (accessed May 24, 2019).

21. Medicines and Healthcare products Regulatory Agency. The Yellow Card Scheme: guidance for healthcare professionals, patients and the public-GOV.UK (2019). Available online at: https://www.gov.uk/guidance/the-yellow-cardscheme-guidance-for-healthcare-professionals (accessed May 24, 2019).

22. Kumar S, Costello AJ, Colman PG. Fournier's gangrene in a man on empagliflozin for treatment of Type 2 diabetes. Diabet Med. (2017) 34:16468. doi: $10.1111 /$ dme. 13508

23. Cecilia-Chi W L-TS. Fournier's syndrome: a life threatening complication of SGLT2 inhibition in poorly controlled diabetes mellitus. It Annu Sci Meet Aust Diabetes Educ Assoc Aust Diabetes Soc. Westmead, NSW: Westmead Hospital (2016).

24. Rodler S, Weig T, Finkenzeller C, Stief C, and Staehler M. Fournier's gangrene under sodium-glucose cotransporter 2 inhibitor therapy as a life-threatening adverse event: a case report and review of the literature. Cureus (2019) 11:e5778. doi: 10.7759/cureus.5778

25. Bersoff-Matcha SJ, Chamberlain C, Cao C, Kortepeter C, Chong WH. Fournier Gangrene Associated With Sodium-Glucose Cotransporter-2 Inhibitors. Ann Intern Med. (2019) 170:764. doi: 10.7326/M19-0085

26. Medicines and Healthcare Products Regulatory Agency. Drug Safety Update. Vol. 12. London: Medicines and Healthcare Products Regulatory Agency (2019).

27. IQVIA. IQVIA, Total Patient Tracker (TPT) Database. Durham, NS IQVIA (2017).

28. Diabetes UK. Diabetes UK Facts and Figures. Diabetes.org (2017). Available online at: https://www.diabetes.org.uk/professionals/position-statementsreports/statistics (accessed May 24, 2019).

29. Chen SL, Jackson SL, Boyko EJ. Diabetes mellitus and urinary tract infection: epidemiology, pathogenesis and proposed studies in animal models. J Urol. (2009) 182:S51-6. doi: 10.1016/j.juro.2009.07.090

30. John LB, Francisco TMSB. Urinary Tract Infections (UTI) in Diabetes Mellitus. Medscape Boston, MA: Harvard Medical School (2017).

31. Ueda P, Svanström H, Melbye M, Eliasson B, Svensson A-M, Franzén $\mathrm{S}$, et al. Sodium glucose cotransporter 2 inhibitors and risk of serious adverse events: nationwide register based cohort study. BMJ. (2018) 363:k4365. doi: 10.1136/bmj.k4365

32. Kmietowicz Z. SGLT2 inhibitors for diabetes are linked to increased risk of lower limb amputation. BMJ. (2018) 363:k4828. doi: 10.1136/bmj.k4828

33. International Conference on Harmonisation of Technical Requirements for Registration of Pharmaceuticals for Human Use the Extent of Population Exposure to Assess Clinical Safety for Drugs Intended for Long-Term Treatment of Non-Life-Threatening Conditions E1 Current Step 4 Version (1994). Available online at: http://www.ich.org/fileadmin/Public_Web_Site/ ICH_Products/Guidelines/Efficacy/E1/Step4/E1_Guideline.pdf (accessed May 24, 2019).

34. ICH Topic E 1 Population Exposure: The Extent of Population Exposure to Assess Clinical Safety Step 5 Note for Guidance on Population Exposure: The Extent of Population Exposure to Assess Clinical Safety (1995). Available online at: http://www.emea.eu.int (accessed May 24, 2019).

35. Kirkwood BR, Sterne JAC. Essentials of Medical Statistics. 2nd editio. Malden, MA: Blackwell Science (2003), 487-92.

36. HMRA. SGLT2 Inhibitors: Reports of Fournier's Gangrene (Necrotising fasciitis of the Genitalia or Perineum)-GOV.UK (2019). Available online at: https:// www.gov.uk/drug-safety-update/sglt2-inhibitors-reports-of-fournier-sgangrene-necrotising-fasciitis-of-the-genitalia-or-perineum (accessed May 24, 2019)

Conflict of Interest: The authors declare that the research was conducted in the absence of any commercial or financial relationships that could be construed as a potential conflict of interest.

Publisher's Note: All claims expressed in this article are solely those of the authors and do not necessarily represent those of their affiliated organizations, or those of the publisher, the editors and the reviewers. Any product that may be evaluated in this article, or claim that may be made by its manufacturer, is not guaranteed or endorsed by the publisher.

Copyright (C) 2021 Taylor, Asmar, Mandal, Tridente, Hardy and Shokrollahi. This is an open-access article distributed under the terms of the Creative Commons Attribution License (CC BY). The use, distribution or reproduction in other forums is permitted, provided the original author(s) and the copyright owner(s) are credited and that the original publication in this journal is cited, in accordance with accepted academic practice. No use, distribution or reproduction is permitted which does not comply with these terms. 